\title{
Relações Familiares e Comportamento Alimentar ${ }^{1}$
}

\author{
Helena Martins Coelho \\ António Prazo Pires \\ Instituto de Psicologia Aplicada - Instituto Universitário
}

\begin{abstract}
RESUMO - Explorou-se a influência das relações familiares no comportamento alimentar das crianças e no desenvolvimento do excesso de peso. Participaram 147 crianças de todas as classes de peso, com idades compreendidas entre os 8 e os 12 anos e respetivas famílias. Às crianças foi aplicada a escala das Relações Familiares do Family Environmemt Scale (FES) e ao principal cuidador o Child Eating Behaviour Questionnaire (CEBQ). Os resultados indicam que o Índice de Massa Corporal (IMC) dos pais é, por si só, um fraco preditor do estatuto de peso dos filhos. Em famílias mais disfuncionais, os filhos têm comportamentos alimentares mais orientados para a atração pela comida, independentemente da classe de peso dos pais
\end{abstract}

Palavras-chave: relação familiar, comportamento alimentar, excesso de peso

\section{Family Relationship and Eating Behaviors}

\begin{abstract}
We explored the influence of family environment, and in particular, the influence of the type of relations established between family members on children's eating behavior and their overweight. A sample of 147 children, with ages between 8 and 12, of all weight classes, completed the Family Relationship subscale of the Family Environmental Scale (FES). Their main caretaker completed the Child Eating Behavior Questionnaire (CEBQ). Parents' Body Mass Index (BMI), by itself, was found to be a weak predictor of children's weight. Family relationship emerged consistently as highly determinant on children's eating behaviors and in families with dysfunctional structures children manifested a greater tendency to behaviors related to attraction to food, regardless of their parent's weight.
\end{abstract}

Keywords: family relationship, eating behaviors, overweight

Muitos autores associam a etiologia da obesidade e do excesso de peso nas crianças a situações, majoritariamente, relacionadas com um estilo de vida sedentário, a erros alimentares e a fatores genéticos (Anderson \& Butcher, 2006; Spruijt-Metz, 2011). Contudo, apesar das campanhas de sensibilização e das medidas implementadas para combater o problema, a prevalência de crianças obesas continua a aumentar significativamente (International Obesity Task Force, 2005; World Health Organization, 2011). Estando a etiopatologia da obesidade associada a múltiplos fatores e comorbidades (Thompson \& Heinberg, 2009) seria esperado que as conclusões encontradas na revisão de literatura não fossem convergentes nem totalmente claras. Os vários autores apontam para a necessidade de estudos que explorem a relação entre os fatores internos e externos ao indivíduo (Byrne, Wake, Blumberg, \& Dibley, 2008, Spruijt-Metz, 2011) e a forma como esses fatores interagem na variação do estatuto de peso das crianças.

Os estudos sobre a influência da predisposição genética no desenvolvimento do excesso de peso indicam que esse fator será um forte preditor do problema (Whitaker, Wright, Pepe, Seidel, \& Dietz,1997). No entanto, nas últimas décadas tem-se verificado um aumento da prevalência do excesso de

1 Este artigo foi realizado no âmbito da Dissertação de Mestrado Integrado em Psicologia Clínica pelo primeiro autor, com coautoria do segundo autor, no Instituto Superior de Psicologia Aplicada no ano de 2012.

2 Endereço para correspondência: Alameda António Sérgio, 1, 13 Esq. Lisboa, Portugal. CEP 1750-031.E-mail: coelhohmsm@gmail.com peso em crianças relativamente estáveis do ponto de vista genético, ou seja, filhos de pais não obesos e sem antecedentes familiares de obesidade (Brazão \& Santos, 2010; Ebbeling, 2002).

Tendo em conta que os fatores genéticos por si só parecem não ser totalmente explicativos, outros investigadores, têm se dedicado ao estudo do excesso de peso e da obesidade procurando conhecer as relações entre os fatores biológicos, psicológicos e sociais (Crothers, Kehle, Bray, \& Theodore, 2009).

Sabe-se da literatura que o funcionamento familiar, tem uma forte influência na alimentação das crianças (Rossi, Moreira \& Rauen, 2008; Savage, Fisher, \& Birch, 2007; Rhee, 2008; Viana, Santos, \& Guimarães, 2008). Parece ser consonante na comunidade científica que a família será o principal mediador social no desenvolvimento e prevenção da obesidade, quer seja pelo estímulo para a prática de atividades físicas, pela oferta acertada de alimentos e modelagem de comportamentos, quer seja pelo ajustamento emocional face à comida (Epstein, 2003).

Sendo através da família que a criança vive as primeiras experiências afetivas e relacionais, não será difícil perceber a sua importância no cuidado e no desenvolvimento saudável da criança (Repetti, Taylor, \& Seeman, 2002). Apesar de não o expressar claramente, a criança parece ter a perceção de que é na família que se dá a elaboração dos sentimentos que estarão na base da formação da sua individualidade (Carvalho, Moreira, \& Rabinovich, 2010). Nas famílias em que as relações são pautadas pelo afeto, pela disponibilidade, pela 
partilha e pela promoção da autonomia, as crianças aprendem a se sentirem seguras e acarinhadas, ficando disponíveis para adquirir comportamentos que lhes vão proporcionar bem-estar físico e emocional (McPhie et al, 2011).

A literatura aponta, ainda. uma estreita ligação entre a obesidade e as questões afetivas e relacionais (Faith, Allison, \& Geliebter, 1997) em que a comida passa a ter uma função reparadora e um simbolismo objetal afetivo (Hamburger, 1951; Levitan \& Davis 2010).

Por outro lado, os estudos sobre a eficácia de tratamentos de perda de peso em crianças, revelaram-se mais positivos quando conjuntamente foram usadas técnicas que levaram ao aumento da coesão e à diminuição do conflito percecionado nas relações familiares (Kitzman-Ulrich et al. 2010). Segundo Beck e Terry (1985), Zeller et al. (2007) e Hasenboehler, Munsch, Meyer, Kappler e Vögele, (2009), as crianças obesas caracterizam o seu ambiente familiar como sendo pouco coeso, pouco estruturado e com valores elevados ao nível do conflito, sendo sugerida uma maior disfuncionalidade familiar.

Outros estudos (eg. Berkowitz et al, 2010; Johannsen, Johannsen, \& Specker, 2006) apontam a cópia dos comportamentos dos pais como sendo significativo na aquisição, pela criança, de comportamentos alimentares que levam ao aumento de peso. A ideia da influência do comportamento parental será mais vincada em particular quando a mãe é obesa (Kral \& Rauh, 2010), estando associado um maior risco de obesidade infantil à obesidade materna, independentemente do sexo (Whitaker et al, 1997). Sendo a mãe, tradicionalmente, a principal cuidadora (Viana, Candeias, Rego, \& Silva, 2009), esta influenciará o estilo alimentar dos filhos. Segundo esses autores, o estilo alimentar diz respeito à categorização dos comportamentos alimentares e poderá ser compreendido tendo por base a reatividade aos estímulos externos dos alimentos (teoria da externalidade), ao controlo da quantidade de comida ingerida (teoria da restrição) e a questões emocionais (teoria psicossomática).

De um modo geral, o comportamento alimentar das crianças com excesso de peso está majoritariamente associado à atração pela comida e se traduz em termos de sobre-ingestão emocional, na falta de controlo na resposta à saciedade e no elevado prazer na comida (Viana, Santos, \& Guimarães, 2008). Por outro lado, a dinâmica familiar, aqui entendida como uma rede relacional que se estabelece entre os vários elementos que constituem o agregado familiar, será determinante em termos de comportamentos (Santos \& Fontaine, 1995) e, como tal, será um bom preditor da obesidade infantil.

O comportamento alimentar e o funcionamento familiar têm sido analisados em diversos estudos sobre as problemáticas alimentares em idades pediátricas (Kitzman-Ulrich et al 2010; Rhee, 2008; Viana et al., 2008; Zeller et al 2007). Contudo, não existem estudos que avaliem a interação das relações familiares e do comportamento alimentar no ganho de peso das crianças.

Será o comportamento alimentar e o estilo de vida das famílias o principal "motor" para o ganho de peso? Ou, serão as relações familiares, o solo afetivo, equilibrante e estruturante das funções da criança, incluído o prazer em se alimentar de forma saudável? De que forma esses fatores interagem?
Assim, é objetivo deste estudo explorar a influência das relações familiares, em particular no que diz respeito ao conflito, à expressividade e à coesão familiar percecionada pelas crianças, no seu comportamento alimentar e qual o impacto da interação desses fatores no ganho de peso das mesmas. Pretende-se ainda avaliar se um comportamento alimentar das crianças mais orientado para a atração pela comida é mais significativo em famílias pouco funcionais; esperando-se que a relação familiar seja, só por si, um forte preditor do comportamento alimentar dos filhos, independentemente do estatuto de peso dos pais.

\section{Método}

\section{Participantes}

A amostra não probabilística é constituída por 147 crianças, com idades compreendidas entre os 8 e os 12 anos, e respetivas famílias. Foram incluídas crianças de ambos os sexos, num total de 36 meninos e 111 meninas, em que $27 \%$ frequentam o ensino público e $73 \%$ o ensino privado e foi recolhida em escolas do $1^{\circ}$ e $2^{\circ}$ ciclos da zona da grande Lisboa.

Cerca de $8 \%$ das crianças da amostra são obesas, $21 \%$ tem excesso de peso, $61 \%$ tem peso normal e $21 \%$ tem peso abaixo do normal, para a estatura/idade.

Relativamente aos pais, $22 \%$ das mães têm excesso de peso e $7 \%$ são moderadamente obesas, enquanto que os pais apresentam uma maior percentagem de excesso de peso $(40 \%)$.

A amostra é majoritariamente constituída por famílias com estrutura biparental e em situação de $1^{\circ}$ casamento ou união de facto.

\section{Instrumentos}

O Child Eating Behaviour Questionnaire (CEBQ) foi desenvolvido por Wardle, Guthrie, Sanderson e Rapoport (2001) para estudar o estilo alimentar das crianças e jovens no contexto da obesidade. Trata-se de um questionário de auto-prenchimento, dirigido ao cuidador, composto por 35 itens distribuídos por oito dimensões: Resposta à saciedade (SR), Ingestão lenta (SE), Seletividade (FF), Resposta à comida (FR), Prazer em comer (EF), Desejo de bebida (DD), Sobre-ingestão emocional (EOE), Sub-ingestão emocional (EUE), quantificados numa escala de cinco pontos do tipo Likert, desde 1 (Nunca) a 5 (Sempre). O Prazer na comida e a Resposta à comida avaliam a resposta aos estímulos externos dos alimentos (cor, aspecto, cheiro, etc). A Resposta à saciedade remete para a sensibilidade interna quanto à sensação de saciedade e auxilia no controle alimentar. A Selectividade e a Ingestão lenta, que avaliam o desinteresse pele comida, são habitualmente mais altos à medida que o peso baixa. A Sub-ingestão e a Sobre-ingestão remetem para a reatividade emocional à comida, estando relacionados com fatores internos do sujeito. O Desejo de Bebida está associado à atração pela ingestão de bebidas calóricas. 
A adaptação à população portuguesa foi feita por Viana e Sinde (2008), em uma amostra de 249 crianças com idades compreendidas entre os 3 e os 13 anos e respectivas mães. Tal como na escala original, o instrumento aferido mostrou ter boas capacidades psicométricas, com valores do alfa de Cronbach compreendidos entre 0,70 (sub-escala EUE) e 0,89 (sub-escala EF). No presente estudo as escalas também apresentaram valores considerados aceitáveis para as ciências humanas (Maroco \& Garcia-Marquez, 2006), apesar de serem ligeiramente mais baixos que os que se verificaram no estudo de aferição. À sub-escala Selectividade (FF), que numa primeira medida apenas possuía um alfa de 0,36 , foram retirados os três itens que menos contribuíam para a consistência interna da escala, tendo-se obtido um valor bastante aceitável $(0,64)$. Os valores de alfa do CEBQ variam, neste estudo, entre 0,64 (FF) e 0,91 (DD). O alfa global da escala é de 0,66.

Segundo Viana e Sinde (2008), as oito sub-escalas que constituem o instrumento podem ser categorizadas em dois grupos: o grupo atração pela comida, que engloba as escalas EF, FR, EOE e DD; e o grupo evitamento da comida que diz respeito às escalas SR, SE, FF e EUE.

O Family Environment Scale (FES) foi desenvolvido por Moos e Moos (1986) com o objetivo de avaliar as perceções pessoais no contexto psicossocial da família, tendo subjacente uma perspectiva sistêmica do funcionamento familiar. A escala é composta por 10 dimensões específicas e três dimensões conceptuais gerais: o Crescimento Pessoal, a Manutenção do Sistema e as Relações Familiares, em que esta última diz respeito aos laços afetivos e emocionais que emergem das interações entre os membros da família e que se refletem em termos de coesão, expressividade e conflito (Moss, 1990).

A avaliação da dinâmica familiar é operacionalizada através de um questionário de auto-preenchimento, que permite avaliar individualmente, ou de forma conjunta, as dimensões gerais e as dimensões específicas (Moos \& Moss, 1986).

Atendendo ao objetivo deste estudo, foi utilizada apenas a escala das Relações Familiares na forma R, que mede a percepção que o sujeito tem do ambiente da sua família nuclear. A referida escala foi aferida à população portuguesa por Matos e Fontaine em 1992, numa população de alunos do ensino básico, com idades compreendidas entre os $8 \mathrm{e}$ os 14 anos, conforme citado por Santos e Fontaine (1995).

A escala portuguesa é do tipo Likert desde 1 (Discordo totalmente) a 6 pontos (Concordo totalmente) sendo composta por três escalas específicas: coesão, conflito e expressividade. A coesão remete para conceitos como a proximidade relacional, ajuda e apoio mútuo, partilha de interesses e objetivos e interdependência dos membros da família. A sub-escala conflito avalia a percepção da angústia que se reflecte em termos de agressividade, zangas, discussões ou outras situações conflituosas. A sub-escala expressividade pretende avaliar a percepção dos elementos da família quanto ao encorajamento para serem expressos abertamente os sentimentos positivos ou negativos (Moos, 1990; Santos \& Fontaine, 1995).

No presente estudo, a dimensão conflito, apesar de individualmente apresentar um bom alfa, quando analisada em conjunto com as restantes duas escalas, retirou totalmente a consistência interna à escala geral, pelo que se optou por desconsiderá-la da análise. Assim, apenas foram utilizadas as sub-escalas coesão e expressividade que obtiveram um alfa de Cronbach de 0,69 e 0,72 , respectivamente. $\mathrm{O}$ alfa total da escala da Relação Familiar da FES é de 0,88.

\section{Procedimento}

Os questionários foram entregues às famílias e recolhidos através de diretores de turma dos alunos com as idades pretendidas. As famílias tomaram conhecimento do objetivo e dos procedimentos do estudo, tendo sido garantida a confidencialidade dos dados fornecidos. A autorização para a participação na investigação foi dada por escrito, através de Consentimento Informado, tendo sido respeitados os princípios éticos e deontológicos instituídos pela Ordem dos Psicólogos Portugueses

Em Portugal, a Direção Geral de Saúde utiliza, desde 2005, as tabelas de Percentil do NCHS-CDC (National Center for Health Statistics-Center for Disease Control and Prevention) na avaliação da evolução corporal das crianças, conforme circular normativa $\mathrm{n}^{\circ} 05 / \mathrm{DSMIA}(\mathrm{DGS}, 2006$ ). Por essa razão, foram usadas as tabelas de percentis do CDC, e respectivos critérios de corte, na definição do Percentil do Índice de Massa Corporal (PIMC) das crianças. No caso dos pais, a classe de peso foi atribuída através do Índice de Massa Corporal (IMC), calculado através da divisão do peso pelo quadrado da altura. Essa medida antropométrica está devidamente validada, sendo a sua utilização recomendada pela WHO.

Sendo a Regressão Linear (RL) bastante robusta a violações ligeiras da distribuição normal das variáveis e da homogeneidade das variâncias dos grupos (Maroco, 2007), utilizou-se esse teste na análise inferencial. Foram tidos em conta os pressupostos de aplicação da Regressão Linear sendo que, os pressupostos da distribuição normal e homogeneidade dos resíduos foram validados graficamente e o pressuposto da independência dos erros foi validado com a estatística de Durbin-Watson em cada modelo criado. Foi ainda utilizado o Variance Inflation Factor (VIF) para diagnosticar se nenhumas das variáveis independentes estariam correlacionadas entre si, no caso dos modelos de Regressão Linear Múltipla. Foram ainda criados modelos para análise da significância da interação, através da análise conjunta das variáveis diretas e do seu respectivo produto, conforme descrito por Maroco (2007). Foram considerados efeitos estatisticamente significativos para um $p<0,05$. Todas as análises estatísticas foram efetuadas com recurso ao software SPSS (v.19; SPSS Inc., Chicago, IL)

\section{Resultados}

Uma análise ao tipo de associação entre o Índice de Massa Corporal (IMC) e o Percentil do Índice de Massa Corporal (PIMC) permitiu identificar uma correlação positiva e extremamente elevada $(r=0,89)$ entre as variáveis. Por esse motivo, optou-se por utilizar o IMC na análise inferencial da variação do peso nos modelos de regressão linear, em substituição da variável PIMC. 


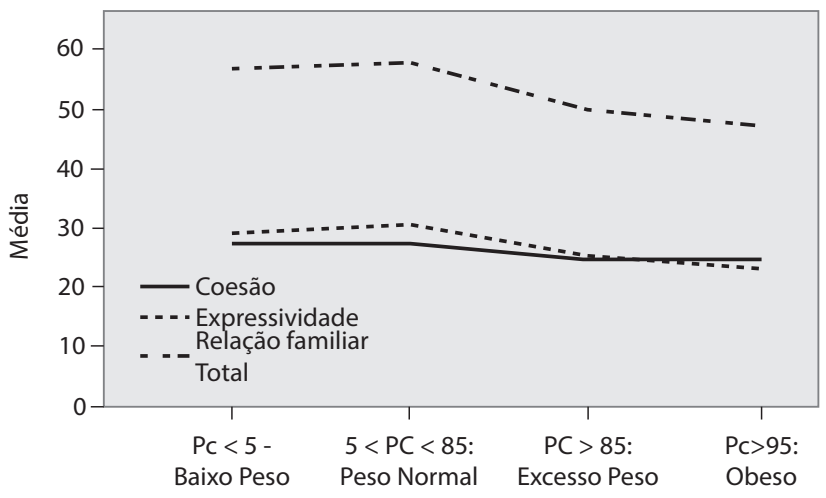

Percentil de IMC

Figura 1. Média das escalas da Relação Familiar (FES), por percentil de IMC dos filhos

\section{Impacto da relação familiar no IMC das crianças}

Como podemos constatar no gráfico da Figura 1, em média, o valor total da escala da relação familiar é mais alto em crianças com peso normal e mais baixo em crianças com excesso de peso e obesas. Quer isso dizer que, as crianças com relações familiares menos coesas e menos expressivas são as que têm, em média, um percentil de IMC mais elevado.

Para avaliar se existem diferenças estatisticamente significativas nas médias dos grupos, foram criados vários modelos de regressão linear, tendo-se constatado que a relação familiar, só por si, explica cerca de 34\% da variação do IMC das crianças $\left(\mathrm{R}^{2} \mathrm{a}=0,34\right)$. Para além disso, verifica-se a influência negativa $(\beta<0)$ de todos os domínios da relação familiar no IMC, ou seja, quanto mais baixa for a coesão e a expressividade, mais alto é o IMC das crianças. A sub-escala que mais negativamente contribui é a da expressividade $(\beta=-0,41 ; t=-5,09 ; p<0,001)$. Relativamente ao modelo que analisou o efeito da interação das relações familiares com o IMC dos pais, no IMC dos filhos, este é ligeiramente mais preditivo $\left(\mathrm{R}^{2} \mathrm{a}=0,40\right)$, no entanto, a relação familiar continua a ser o fator que mais faz variar o IMC das crianças $(\beta=-0,49 ; t=-7,22 ; p<0,001)$. De salientar ainda que, nesse último modelo, o IMC do pai não contribui significativamente para a variação do IMC dos filhos $(\mathrm{t}=0,86 ; p=0,393>0,05)$.

\section{Impacto do comportamento alimentar das crianças no seu IMC}

Como vimos, as oito dimensões que constituem o CEBQ podem ser categorizadas em dois grupos: o grupo atração pela comida e o grupo evitamento da comida. Regra geral, a média das sub-escalas que remetem para a atracão pela comida apresentaram-se superiores nas crianças com PIMC mais elevado. Relativamente ao grupo evitamento da comida, e como seria espectável, as médias mais altas das sub-escalas apresentadas situam-se nas crianças que têm peso baixo.

Para averiguar o efeito do comportamento alimentar na variação do IMC das crianças e perceber quais os fatores que mais contribuem para essa variação, foi então criado um modelo de RL que avaliasse a variância do IMC em função de cada um dos fatores do CEBQ. Verificou-se que as dimensões sub-ingestão emocional, ingestão lenta, prazer na comida, selectividade e desejo de bebida não são significativas para a variância do IMC das crianças da amostra $(p>0,05)$. Adicionalmente, constatou-se que o factor que mais influenciou o modelo foi a resposta à saciedade $(\beta=-0,386)$. Quer isso dizer que as crianças com excesso de peso terão um controlo interno da sensação de "estar cheio" mais deficitário. Como seria esperado, a sobre-ingestão emocional e a resposta à comida contribuem positivamente para um comportamento alimentar direcionado para a apetência para a comida, levando ao aumento do IMC. Nessa análise, o comportamento alimentar explica cerca de $40 \%$ da variância do IMC nas crianças deste estudo.

Pretendeu-se ainda avaliar o impacto da classe de peso dos pais, analisado pelo respetivo IMC, em interação com a variável comportamento alimentar dos filhos, no seu ganho de peso, por sexo. No que diz respeito às meninas, verifica-se que a interação do comportamento alimentar com o IMC da mãe é bastante representativa na variância do IMC dos filhos $(\beta=-0,384 ; t=4,63 ; p<0,001)$. Por outro lado, a análise conjunta com o IMC do pai não se revelou significativa. Em relação aos meninos, é a interação do IMC do pai com o comportamento alimentar dos filhos que se mostrou mais significativo $(\beta=-0,468 ; t=3,28 ; p=0,003)$. Nesse modelo, o IMC das mães não é expressivo para a variância de peso nos meninos. Quando a análise é feita sem diferenciar o sexo das crianças, apenas o IMC da mãe se associa ao comportamento alimentar dos filhos, na variação do seu IMC ( $\beta=-0,339$; $t=4,67 ; p<0,001)$.

Em suma, o IMC dos pais parece atuar como uma variável que potencia o comportamento alimentar das crianças, de tal modo que o IMC da mãe faz aumentar o IMC das filhas e o IMC do pai faz aumentar o IMC dos filhos do sexo masculino. No entanto, no geral é a classe de peso da mãe que mostra ter mais influência na variação de peso dos filhos.

\section{Impacto das relações familiares e do comportamento alimentar no IMC dos filhos}

Após ter sido analisado o efeito das relações familiares e do comportamento alimentar dos filhos no seu ganho de peso, de forma isolada, pretendeu-se de seguida conhecer o efeito conjunto do comportamento alimentar e da relação familiar na variação do peso dos filhos. Recorrendo-se, novamente, à regressão linear para essa análise verificou-se que o modelo é bastante explicativo $\left(\mathrm{R}^{2} \mathrm{a}=0,36 ; \mathrm{F}=41,75\right)$, sendo que a relação familiar é a variável que mais contribuiu para a variância total do IMC dos filhos $(\beta=-0,502 ; t=-6,489 ; p<0,001)$.

Na Figura 2 está representada a média do IMC, em função do comportamento alimentar e da relação familiar. As categorias das variáveis foram definidas com base na respectiva mediana. Como podemos ver, a média do IMC é inversamente proporcional ao evitamento da comida, ou seja, à medida que o evitamento da comida vai diminuindo, vai aumentando o valor do IMC. Salienta-se a acentuada diferença na média do IMC quando a relação familiar é menos 


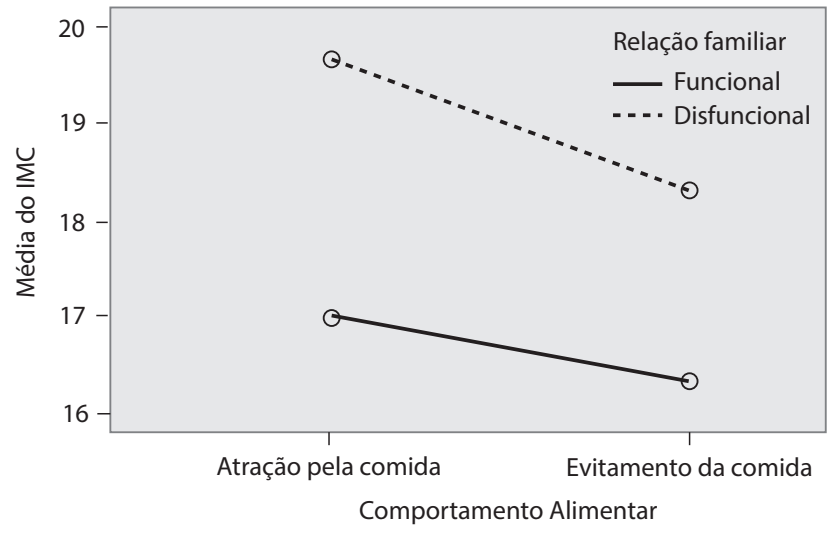

Figura 2. Efeito da interação da relação familiar e do comportamento alimentar no IMC dos filhos

funcional, quer em comportamentos de atração pela comida quer em comportamentos de evitamento, dão nota do efeito de interação dessa variável, que será ainda mais acentuado em comportamentos mais orientados para a comida.

\section{Impacto das relações familiares no comportamento alimentar dos filhos}

Nos pontos anteriores analisamos o efeito das relações familiares e do comportamento alimentar no IMC dos filhos. Neste momento, iremos averiguar se as relações familiares são determinantes para o comportamento alimentar dos filhos, independentemente do estatuto de peso dos pais.

Com esse objetivo, recorremos à análise da variância através de vários modelos de regressão linear. Assim, criou-se num primeiro momento um modelo apenas com as variáveis IMC dos pais, depois um modelo no qual se incluiu a variável relação familiar e, por fim, um modelo simples apenas com a relação familiar.

Como podemos retirar da análise da Tabela 1, o IMC dos pais será por si só um fraco preditor do estilo alimentar dos filhos $\left(\mathrm{R}^{2} \mathrm{a}=0,072\right)$. Quando se introduziu na análise a variável relação familiar, constatou-se que esta produziu um efeito apreciável na variação do comportamento alimentar $(\beta=-0,482 ; t=-6,39 ; p<0,001)$ tornando o modelo mais explicativo $\left(R^{2} a=0,273\right)$. Ainda segundo esta análise diferencial, $o$ IMC do pai não contribui significativamente para o modelo $(p=0,09>0,05 \alpha)$. A significância dessa interação foi confirmada estatisticamente. A suspeita de que a relação familiar teria impacto no comportamento alimentar veio a confirmar-se, na medida em que $26 \%$ da variância do comportamento alimentar é explicado apenas pela relação familiar, valor idêntico ao do modelo de RL, do que se incluiu, simultaneamente, o IMC dos pais. E se atentarmos ao valor de $\beta$ $(-0,516)$, confirmamos que essa variável se apresenta como um preditor significativo do comportamento alimentar. Os resultados levam-nos a inferir que, quanto menos funcional for a relação familiar, mais elevados serão os valores da escala do comportamento alimentar que, como vimos anteriormente, remete para o conceito de apetência pela comida.

Em seguida, averiguou-se quais as dimensões do comportamento alimentar que seriam mais influenciadas pela relação familiar. Nessa análise, todos os modelos se mostraram significativos, tendo-se identificado a resposta à comida (FR) e a resposta à saciedade (SR), como sendo as dimensões nas quais mais se fez sentir o efeito da Relação Familiar. O modelo mais explicativo prevê cerca de 37\% dos comportamentos e o menos explicativo, apenas cerca de $2 \%$ dos comportamentos de selectividade dos alimentos (FF). Salienta-se ainda o significado do efeito negativo da variável relação familiar na resposta à comida, ou seja, quanto menos funcional for a relação familiar, mais elevado será o valor dessa sub-escala. Situação idêntica se passa com as dimensões EOE, EF e DD, isto é, valores menos positivos na relação familiar levam ao aumento da sobre-ingestão emocional, do prazer em comer e do desejo de beber.

A título exploratório, averiguou-se sobre as dimensões da relação familiar que mais contribuíam para o aumento das dimensões indicadas anteriormente, tendo-se concluído que a expressividade mostrou-se estatisticamente significativa para todas as escalas do comportamento alimentar. Um ambiente familiar percepcionado como sendo pouco aberto à expressão de sentimentos e emoções, parece contribuir de forma significativa para um comportamento alimentar mais desadequado nas crianças.

Tabela 1. Resultados dos modelos de regressão linear, sobre o efeito da relação familiar no comportamento alimentar dos filhos, com e sem inclusão do IMC dos pais

\begin{tabular}{|c|c|c|c|c|c|c|}
\hline & \multirow{2}{*}{$\begin{array}{c}\text { Modelo } \\
\mathrm{R}^{2} \mathrm{a}\end{array}$} & \multicolumn{2}{|c|}{ ANOVA } & \multicolumn{3}{|c|}{ Coeficientes } \\
\hline & & $\mathrm{F}$ & Sig & Standard. $\beta$ & $\mathrm{t}$ & Sig \\
\hline IMC do Pai & \multirow{2}{*}{0,072} & \multirow{2}{*}{6,64} & \multirow{2}{*}{0,002} & 0,217 & 2,65 & 0,047 \\
\hline IMC da Mãe & & & & 0,151 & 1,84 & 0,009 \\
\hline IMC do Pai & \multirow{3}{*}{0,273} & \multirow{3}{*}{19,26} & \multirow{3}{*}{0,000} & 0,08 & 0,108 & 0,091 \\
\hline IMC da Mãe & & & & 0,15 & 2,05 & 0,042 \\
\hline Relação Familiar & & & & $-0,482$ & $-6,39$ & 0,000 \\
\hline Relação Familiar & 0,261 & 52,51 & 0,000 & $-0,516$ & $-7,25$ & 0,000 \\
\hline
\end{tabular}




\section{Discussão}

O objetivo geral deste estudo foi explorar a influência das relações familiares no comportamento alimentar das crianças e no seu ganho de peso. Não será difícil de compreender que um estilo de vida menos saudável e uma alimentação mais calórica terá como consequência direta o aumento de peso nas famílias. O que se questiona é se o comportamento alimentar orientado para a comida é uma consequência de um contexto ambiental potencialmente obesogênico, marcado por uma pobreza relacional e afetiva.

Uma das primeiras elações que se retira dos resultados é que, ao contrário de vários estudos encontrados na revisão de literatura e que apontam o excesso de peso dos pais como um forte preditor do excesso de peso dos filhos, o estatuto ponderal dos pais não parece ser um determinante direto do peso dos filhos. Na verdade, quando analisado em conjunto com as relações familiares, apesar de ser significante, o seu impacto na variação do IMC dos filhos foi bastante reduzido. Por outro lado, as crianças que percepcionam um ambiente familiar em que se sentem apoiadas, em que existe um maior envolvimento e uma maior liberdade para expressar o que sentem, são mais normativas em termos de classe de peso. Uma maior funcionalidade familiar relacionou-se positivamente com um estilo de alimentação mais saudável e a comportamentos que remetem para uma ingestão mais controlada. Esses resultados serão indicadores da importância das relações familiares no desenvolvimento de um peso saudável e estão de acordo com os trabalhos de Beck e Terry (1985) e Hasenboehler et al (2009), que remetem para a importância do ambiente familiar no desenvolvimento e na prevalência do excesso de peso. Um ambiente caracterizado pela proximidade relacional, apoio mútuo e capaz de proporcionar à criança a livre expressão de sentimentos, será facilitador de um desenvolvimento físico e psicológico mais salutar (McPhie et al, 2011; Santos \& Fontaine, 1995). Vem ainda dar suporte à concepção de que a resposta aos estímulos dos alimentos será influenciada por questões afetivas e relacionais, reforçando os achados de Zeller et al (2007) e de Kizman-Ulrich et al (2010) quanto à importância de trabalhar as interações familiares nas intervenções terapêuticas em crianças obesas. Não quer isso dizer que os antecedentes de excesso de peso na família, fortemente relacionados na literatura com a predisposição hereditária, não sejam significativos, mas o ambiente familiar onde a criança cresce, poderá, ou não, potenciar o seu desenvolvimento.

Quando analisamos os resultados diferenciados por sexo, e ao contrário do que seria esperado, a classe de peso da mãe apenas interagiu com o comportamento alimentar das meninas. Sendo a mãe, habitualmente, a principal cuidadora dos filhos esperava-se que, tendencialmente, existisse uma influência da conduta alimentar da mãe (Berkowitz et al, 2010), representada pela sua classe de peso, no peso dos filhos, independentemente do sexo, o que não se verificou. Uma possível explicação, fundamentada no modelo de Bronfenbrenner (1999), será o facto da estrutura familiar e dos papéis dos familiares terem vindo a sofrer profundas alterações, em que assistimos a um maior envolvimento paterno nas tarefas domésticas e no cuidado dos filhos. O papel de mãe cuidadora tenderá a ser cada vez mais partilhado pelo pai, tornando pertinente focalizar o estudo do excesso de peso e da obesidade tendo em conta não só o envolvimento materno, mas também o paterno. Por outro lado, e se atentarmos ao facto de existir uma maior associação entre o peso dos pais e dos filhos do mesmo sexo, poderemos ser levados a colocar o cerne da problemática em processos identificatórios secundários. Resultados similares foram encontrados por Perez-Pastor et al. (2009) Segundo esses investigadores, a elevada correlação entre o peso dos pais e o peso dos descendentes do mesmo sexo, será um indicador da influência familiar ou da identificação aos pais, e não da predisposição genética. O presente estudo levanta a necessidade de explorar o significado do envolvimento paternal e do suporte paterno no desenvolvimento do excesso de peso nos filhos, sendo particularmente importante compreender as relações que se estabelecem entre díades do mesmo sexo.

Os resultados evidenciam a predominância do ambiente familiar no ganho de peso das crianças. Sendo o contexto familiar um lugar de partilha de afetos, cuidados e padrões culturais, é pela intersubjectividade que se processa a construção dos processos psicológicos e das características individuais. Essa dinâmica assenta em processos relacionais, como a proximidade, o suporte emocional, o apoio mútuo, a promoção da autonomia, da capacidade de pensar e de expressar as emoções. Tendo-se verificado no presente estudo uma maior incidência de crianças com excesso de peso em famílias pouco funcionais, podemos ser levados a dizer que comer poderá ser para a criança, por um lado, a forma que encontra para se estruturar numa família que sente vazia de afetos, que não a "alimenta" narcisicamente e, por outro, uma forma de usar o corpo como proteção de um ambiente familiar disfuncional.

Relativamente às limitações deste estudo, a primeira consideração a ter na leitura dos resultados prende-se com o próprio processo de amostragem que, sendo por conveniência e portanto não probabilística, devem ser generalizados com cautela.

Outra condicionante está relacionada com o método de recolha de dados, em particular, no que diz respeito à altura e peso das crianças e dos pais que foi auto-indicada. Seria útil que futuros estudos garantissem a recolha da informação através do registo presencial dessas medidas, cuja precisão é crítica para este tipo de análise.

Importa ainda, em investigações futuras, categorizar as características familiares que levam a um funcionamento menos adequado, explorando as relações que se estabelecem entre os vários elementos, de modo a conhecer de forma mais aprofundada a dinâmica das famílias com problemas de obesidade. Conhecer os padrões de interação que se associam à disfuncionalidade da família será fundamental para prover comportamentos saudáveis e estabelecer modelos interventivos e preventivos do desenvolvimento de excesso de peso em idades pediátricas. 


\section{Referências}

Anderson, P. M., \& Butcher, K. F. (2006). Childhood Obesity: Trends and Potential Causes. Future of Children, 16(1), 19-45. Retrieved from http://www.princeton.edu/futureofchildren/ publications/docs/16_01_02.pdf

Beck, S., \& Terry, K. (1985). A comparison of obese and normal-weight families' psychological characteristics. American Journal of Family Therapy, 13(3), 55-59. doi:10.1080/01926188508251264

Berkowitz, R. I., Moore, R. H., Faith, M. S., Stallings, V. A., Krai, T. E., \& Stunkard, A. J. (2010). Identification of an obese eating style in 4-year-old children born at high and low risk for obesity. Obesity, 18(3), 505-512. doi:10.1038/oby.2009.299

Brazão, N., Santos, O. (2010). Transgeracionalidade na obesidade infantil. Endocrinologia, Diabetes \& Obesidade, 4(2), 87-94.

Bronfenbrenner, U. (1999). Environments in developmental perspective: Theoretical and operational models. In S. L. Friedman, T. D. Wachs, S. L. Friedman, \& T. D. Wachs (Eds.), Measuring environment across the life span: Emerging methods and concepts (pp. 3-28). Washington, DC US: American Psychological Association. doi:10.1037/10317-001

Byrne, S., Wake, M., Blumberg, D., \& Dibley, M. (2008). Identifying priority areas for longitudinal research in childhood obesity: Delphi technique survey. International Journal of Pediatric Obesity, 3(2), 120-122. doi:10.1080/17477160701830796

Carvalho, A.M.A., Moreira, L.,V.,C., \& Rabinovich, E., P. (2010). Olhares de crianças sobre a família: um enfoque quantitativo. Psicologia: Teoria e Pesquisa, 26(3), 417-426

Crothers, L. M., Kehle, T. J., Bray, M. A., \& Theodore, L. A. (2009). Correlates and suspected causes of obesity in children. Psychology in the Schools, 46(8), 787-796. doi:10.1002/ pits. 20417

DGS (2006). Circular Normativa $n^{\circ}$ 05/DSMIA de 21/02/2006 da Direção Geral de Saúde. Retrieved from http://www.dgs.pt/

Ebbeling, C. S. (2002). Childhood obesity: public-health crisis, common sense cure. Lancet, 360(9331), 473-482.

Epstein, L. H. (2003). Development of evidence-based treatments for paediatric obesity. In A. E. Kazdin, \& J. R. Weisz (Eds.), Evidence-based psychotherapies for children and adolescents (pp. 374-388). New York: Guilford Press.

Faith, M. S., Allison, D. B., \& Geliebter, A. (1997). Emotional eating and obesity: Theoretical considerations and practical recommendations. In S. Dalton (Ed.), Overweight and weight management: The health professional's guide to understanding and practice (pp. 439-465). Gaithersburg, MD US: Aspen Publishers.

Hamburger, W. W. (1951). Emotional aspects of obesity. Medical Clinics of North America, 35, 483-499.

Hasenboehler, K., Munsch, S., Meyer, A. H., Kappler, C., \& Vögele, C. (2009). Family structure, body mass index, and eating behavior. International Journal of Eating Disorders, 42(4), 332-338. Retrieved from http://web.a.ebscohost.com/ehost/ pdfviewer/pdfviewer?sid=3769e1d1-1c6c-4f6c-8af1-277c26 6f $29 \mathrm{~b} \%$ 040sessionmgr4003\&vid=58\&hid $=4104$

International Obesity Task Force (2005). Obesity in Europe. EU Platform Briefing Paper. Bruxelas: IOTF.
Johannsen, D. L., Johannsen, N. M., \& Specker, B. L. (2006). Influence of Parents' Eating Behaviors and Child Feeding Practices on Children's Weight Status. Obesity, 14(3), 431-439. doi:10.1038/oby.2006.57

Kitzman-Ulrich, H., Wilson, D. K., St. George, S. M., Lawman, H., Segal, M., \& Fairchild, A. (2010). The Integration of a Family Systems Approach for Understanding Youth Obesity, Physical Activity, and Dietary Programs. Clinical Child and Family Psychology Review, 13(3), 231-253.

Kral, T. E., \& Rauh, E. M. (2010). Eating behaviors of children in the context of their family environment. Physiology \& Behavior, doi:10.1016/j.physbeh.2010.04.031

Levitan, R. D., \& Davis, C. (2010). Emotions and Eating Behaviour: Implications for the Current Obesity Epidemic. University of Toronto Quarterly, 79(2), 783-799. doi:10.3138/UTQ.79.2.783

Maroco, J. \& Garcia-Marquez, T. (2006). Qual a fiabilidade do alfa de Cronbach? Questões antigas e soluções modernas. Laboratório de Psicologia, 4(1), 65-90. Lisboa: ISPA.

Maroco, J. (2007). Análise Estatística - Com Utilização do SPSS. Lisboa: Edições Sílabo.

McPhie, S., Skouteris, H., McCabe, M., Ricciardelli, L. A., Milgrom, J., Baur, L. A., \& Dell'Aquila, D. (2011). Maternal correlates of preschool child eating behaviours and body mass index: a cross-sectional study. International Journal of Paediatric Obesity, 6(5/6), 476-480. doi:10.3109/17477166. 2011.598937

Moos, R. H \& Moos, B. S. (1986). Family Environment Scale. Manual. Palo Alto, CA: Consulting Psychologists Press.

Moos, R. H. (1990). Conceptual and empirical approaches to developing family-based assessment procedures: Resolving the case of the Family Environment Scale. Family Process, 29(2), 199-208. doi:10.1111/j.1545-5300.1990.00199.x

Perez-Pastor, E. M., Metcalf, B. S., Hosking, J. J., Jeffery, A. N., Voss, L. D., \& Wilkin, T. J. (2009). Assortative weight gain in mother-daughter and father-son pairs: an emerging source of childhood obesity. Longitudinal study of trios (EarlyBird 43). International Journal of Obesity, 33(7), 727-735. doi:10.1038/ ijo.2009.76

Repetti, R., Taylor, S., \& Seeman, T. (2002). Risky families: family social environments and the mental and physical health of offspring. Psychological Bulletin, 128 (2), 330-366. Retrieved from http://repettilab.psych.ucla.edu/1.htm

Rhee, K. (2008). Childhood overweight and the relationship between parent behaviors, parenting style, and family functioning. Annals of the American Academy of Political and Social Science, 615(1), 12-37. doi:10.1177/0002716207308400

Rossi A., Moreira E., Rauen M. (2008). Determinantes do comportamento alimentar: uma revisão com enfoque na família. Revista de Nutrição, 21, 739-748. Retrieved from http://dx.doi.org/10.1590/S1415-52732008000600012

Santos, M., \& Fontaine, A. M. (1995). Avaliação do ambiente familiar por crianças e pré-adolescentes: alguns aspectos da adaptação da FES de Moos \& Moos. In L. S. Almeida \& I. S. Ribeiro (Eds.). Avaliação Psicológica: Formas e Contextos, vol. III (421-430). Braga: APPORT. 
Savage, J. S., Fisher, J., \& Birch, L. L. (2007). Parental Influence on Eating Behavior: Conception to Adolescence. Journal of Law, Medicine \& Ethics, 35(1), 22-34. doi:10.1111/j.1748720X.2007.00111.x

Spruijt-Metz, D. (2011). Etiology, Treatment, and Prevention of Obesity in Childhood and Adolescence: A Decade in Review. Journal of Research on Adolescence, 21(1), 129-152. Retrieved from http://web.a.ebscohost.com/ehost/pdfviewer/ pdfviewer?vid=59\&sid=3769e1d1-1c6c-4f6c-8af1-277c266f 29b9\%40sessionmgr4003\&hid $=4104$

Thompson, J., \& Heinberg, L. J. (2009). Future directions in pediatric obesity. In L. J. Heinberg, J. Thompson, L. J. Heinberg, J. Thompson (Eds.), Obesity in youth: Causes, consequences, and cures (pp. 203-208). American Psychological Association. doi:10.1037/11868-010

Viana, V., Santos, P. L., \& Guimarães, M. J. (2008). Comportamento e hábitos alimentares em crianças e jovens: uma revisão de literatura. Psicologia, Saúde \& Doenças, 9(2), 209-231.

Viana, V., \& Sinde, S. (2008). O comportamento alimentar em crianças: estudo de validação de um questionário numa amostra portuguesa (CEBQ). Análise Psicológica, 1(XXVI), 111-120

Viana, V., Candeias, L., Rego, C., \& Silva, C. (2009). Comportamento alimentar em crianças e controlo parental: uma revisão de literatura. Alimentação Humana, 15(1), 9-15.
Wardle, J., Guthrie, C.A., Sanderson, S., \& Rapoport, L. (2001). Development of the Children's Eating Behaviour Questionnaire. Journal of Child Psychology and Psychiatry, 42, 963970.

Whitaker, R. C., Wright, J. A., Pepe, M. S., Seidel, K. D., \& Dietz, W. H. (1997). Predicting Obesity in Young Adulthood from Childhood and Parental Obesity. New England Journal of Medicine, 337(13), 869-873. doi:10.1056/ NEJM199709253371301

World Health Organization (2011). Obesity and overweight. Fact sheet $\mathrm{N}^{\circ} 311$. Geneva: WHO

Zeller, M., H., Reiter-Purtill, J., Modi, A. C., Gutzwiller, J., Vannatta. K. \& Davies, W. H.(2007). Controlled Study of Critical Parent and Family Factors in the Obesigenic Environment. Obesity, 15(1), 126-136. Retrieved from http://www.nature.com/oby/ journal/v15/n1/abs/oby2007517a.html

Recebido em 27.06.2012

Primeira decisão editorial em 11.06.2013

Versão final em 09.07.2013

Aceito em 07.08.2013 